\title{
Research Paper: Translation, Cultural Adaptation, and Validation of Psychometric Properties of the Parent Section of Parent-Child Interaction Coding System
}

\author{
Sanaz Dehghani ${ }^{1}$ (D) Fariba Yadegari $^{1^{*}}$ (D), Atieh Ashtari1 ${ }^{1}$ (D), Akbar Biglarian² ${ }^{2}$ (D) \\ 1. Department of Speech Therapy, University of Social Welfare and Rehabilitation Sciences, Tehran, Iran \\ 2. Department of Biostatistics, Pediatric Neurorehabilitation Research Center, University of Social Welfare \& Rehabilitation Sciences, Tehran, Iran.
}

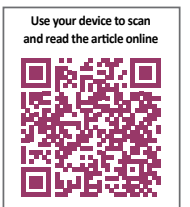

Citation: Dehghani S, Yadegari F, Ashtari A, Biglarian A. Translation, Cultural Adaptation, and Validation of Psychometric Properties of the Parent Section of Parent-Child Interaction Coding System. Iranian Rehabilitation Journal. 2021; 19(3):241-250. http://dx.doi.org/10.32598/irj.19.3.1129.1

http://dx.doi.org/10.32598/irj.19.3.1129.1

Article info:

Received: 07 May 2020

Accepted: 06 Jul 2021

Available Online: 01 Sep 2021

\section{Keywords:}

Parent-child interaction, Adaptation, Validity, Culture, Language development

\section{A B STRACT}

Objectives: Most of the tests regarding parent-child interaction have dealt with this issue from the emotional-behavioral point of view; however, studying these interactions from a combined verbal-behavioral aspect requires a tool consistent with Iranian culture. Parent-Child interaction coding system- $3^{\text {rd }}$ edition (DPICS-III, 2009) is allotted to evaluate parent-child interaction. This study was conducted on mothers of 3- to 6-year-old children to translate, adapt to the Persian language, and determine the validity and reliability of the parent section of this test.

Methods: This research was descriptive-analytic with a non-experimental design. This test was adapted to the Persian language, and its face validity was examined to clarify the items. Moreover, the correlation between live and video observations of 10 mothers and children was evaluated to determine the validity of observations. Later on, the same rater reviewed the videos of 10 mothers - recorded for the correlation between live and video observationsagain within a week for intra-rater reliability and verify the credibility of the rater. Moreover, the Spearman and interclass correlation coefficients were used to determine inter-rater reliability. Data analysis was done by calculating ICC, the Pearson correlation coefficient, and the Spearman correlation coefficient.

Results: The translation process led to an appropriate version of the test in Persian language. The Pearson and Spearman correlation coefficients were used for analysis. The correlation between live observation and video was significant $(\mathrm{P}<0.05,1-0.74=$ spearman and $\mathrm{P}>0.05$, $0.99-0.83=$ Pearson $)$. The reliability of intra-rater was also high $(\mathrm{P}<0.05,1-0.89=$ spearman and $\mathrm{P}>0.05,0.99-0.87=$ Pearson). Furthermore, inter-rater reliability was also high $(\mathrm{P}<0.05$, 0.99-0.81=spearman and $\mathrm{P}<0.05,1-0.79=\mathrm{ICC})$.

Discussion: According to the study results, the parent section of DPICS is applicable in the Persian language with appropriate validity and reliability. This applicable test is either as live observation or video recording, with no differences

* Corresponding Author:

Fariba Yadegari, MSC.

Address: Department of Speech Therapy, University of Social Welfare and Rehabilitation Sciences, Tehran, Iran.

Tel: +98 (21) 22180043

E-mail:faribayadegari@yahoo.com 


\section{Highlights}

- A suitable tool in the Persian language was prepared to evaluate parent-child interactions in children aged 3-6 years.

- Using this test, a 2-way coding system of the child's parent interaction in clinical and research activities in Persian can be considered.

\section{Plain Language Summary}

The pattern of parents' interaction with their children has a significant effect on their natural development. Besides, the essential elements of language are made available to them through the social interactions of parents and children. For this reason, examining the quality of parent-child interaction is essential. Considering that most parent-child interactive tests assess the quality of parent-child interaction unilaterally, it is necessary to use a comprehensive test to assess the quality of parent and child interaction bilaterally. Because of the limited access of Persian language speech therapists to such a test, we decided to examine the validity and reliability of the dyadic parent-child interaction coding system in Persian.

\section{Introduction}

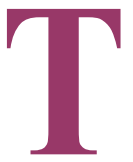

he child's development and growth result from the continuous and dynamic exchange of biological, genetic, and environmental characteristics. The parentchild relationship is one of the critical factors affecting the psychosocial development of children. It is so significant that the quality of parent-child relationships in the early years of growth sets the foundation for a child's cognitive, social, and emotional status in the future [1]. From infancy onwards, parent-child interactions provide the primary social learning context. Emotion regulation and recognition, referencing, gaze following, gesturing, and communication are just some of the social-cognitive and socio-emotional processes first visible in parent-child interactions [2]. Various studies have shown that interactive patterns of mother-child have a relationship with self-incapacitation, eating disorders, anxiety, and psychosomatic problems in children [3-5]. It has also been stated that essential elements of language are made available to the children through social interactions between parents and children $[6,7]$.

On the other hand, teaching and research on the interaction of parents with children facilitate speech therapy sessions and can also identify parent's behavioral problems while interacting with their child, which also contributes to facilitating the child's talk development [8]. Cologon et al. claimed that the parent's response to their children would be effective in communicative skills and, consequently, developing their child's language [9]. Almost the same result was obtained by Brady and Warren and Konza et al., who showed the importance of this subject $[10,11]$. More importantly, the level of children's interaction with their environment is strongly influenced by parental prosody, which should be considered as an important factor in parent-child interaction [12].

To investigate the interactive methods of parents, trainers, and therapists in contact with children, as well as the advancement of pediatric treatment by the therapist, we need a tool that measures verbal and behavioral features simultaneously. Parent-Child Interaction Coding System (DPICS) is a well-known tool to evaluate the effect of parent-child interaction on the psychosocial development of children. In 1981, Eyberg and Robinson standardized this test for the first time, codified by live observation [13]. Subsequently, to complete the DPICS test (second edition), Besmer and Eyberg encoded its subsets [14].

They examined 20 parent-child interactions in two game situations: child-centered and parent-centered. The reliability values in their parent and child classes were obtained using the kappa inter-rater method, from 0.29 to 0.89 and from 0.24 to 0.95 , respectively. Also, they determined the reliability between normal and Oppositional Defiant Disorder (ODD) mother-child, from 0.46 to 0.86 in parent classes and from 0.33 to 0.87 in children classes using the kappa method. In another study by Brestan et al. on 60 father-child pairs, in two groups of normal and with ODD, the reliability of classes of the child was from 0.8 to 0.95 and from 0.26 to 0.97 for the father using kappa inter-rater agreement [15].

It should be noted that language and interaction between parents and children strongly depend on the nature of culture $[16,17]$. On the other hand, most tests for studying the parent-child interaction in speech and language therapy (such as DPICS) have been developed 
using Western approaches, which are not consistent with Iranian communication, verbal, and cultural structures.

To the best of our knowledge, DPICS tests on Iranian parents and children have not been performed previously. Therefore, this study was conducted with the following objectives: 1) translation into and adaptation of this test to the Persian language, 2) review of the face validity of the test, 3) examining the correlation between live and video observations, 4) examining the intra-rater reliability, and 5) examining inter-rater reliability.

\section{Materials and Methods}

\section{Survey tools}

The third edition of the DPICS test (DPICS-III) is an observational and behavioral system designed to evaluate the quality of social engagements of parents and their 3-6 years old children. DPICS-III includes two classes: standard class and complementary class. Both classes have subclasses of parent and child, and each subclass includes several behavioral and vocal subclasses. These behavioral and vocal subsets in standard classes include verbalization, vocalization, physical, and response categories. In the standard class of the parent part, the verbalization part has items such as negative talk, direct and indirect command, labeled and non-labeled praise, information question, descriptive/reflective question, reflective statement, behavioral description, and neutral talk. The physical category parts include positive touch and negative touch. The response and vocalization categories do not have any subset. This test is performed in three positions for 25 minutes; the first DPICS situation is called child-led play (CLP; 10 minutes with considering warm-up time) and the second position is parent-led play (PLP; 10 minutes), and the third is cleaning up the tools (CU; 5 minutes). This test can be performed as live and videotape.

\section{Study participants}

The study participants were 47 mothers of 3-6 years old Persian children. Their children had no speech problems, were monolingual, and also the mothers themselves were mentally healthy. The families were from the middle class of society. Five kindergartens were randomly selected from different regions of Tehran City, Iran (one kindergarten from each zone). In each kindergarten, 15 children of 3-6 years old were chosen as available samples that the sample size of these kindergartens reached 75. After the constitution of justification sessions and obtaining the consent letter from the parents of selected children, the survey was started. Later on, the samples size was reduced to 56 individuals because of different reasons such as illness and non-cooperation of the child and or absence of the parent for continuing the process.

\section{Method of survey performance}

After getting permission from the test designer for the test evaluation in Iran, the guidelines of test performance and its tasks were translated into Persian.

The Biton method was used for the cultural adaptation of this test. This method includes 5 stages of translational and discussion, which are used to eliminate cultural conflict in tests [18]. Also, experts evaluated the test for formal features and all factors of evaluation of assignment of target skills, including the manner of performing the tasks and its physical conditions from the behavioral and linguistic points. After paying attention to alterations accomplished in test items and then the guideline by experts' evaluations, the final form of the test was provided for the main performance.

Afterward, after approval of the Welfare Organization and coordination with the kindergarten manager, the study was started in kindergartens. First, the physical conditions and required tools for test performance mentioned in guidelines were provided to perform the test. In the first stage, coding of behaviors was performed in live and videotape from 10 families. The mother-child engagement was recorded by the first researcher using a video camera (SONY CYBERSHOT WX 80, Japan). In the next stage, the recorded engagement of the samples was observed by the first researcher. The recording was done based on test guidelines, and all 10 mothers were recorded in the provided forms.

Because there was a high correlation between the coded behaviors in the live method and recorded video, the expertise and skill of the first evaluator were confirmed, and the survey reached the next stage. In this stage, the arrangements were made to allow the second evaluator to decide on the test coding. These arrangements were studying the quality of coding, checking several videos, and holding question and answer sessions with the first evaluator. This process lasted two months. Then, the two evaluators participated in the session and coded the 56 behaviors of mothers by the live observation. The scoring in this system was performed based on the observed frequency of behaviors. In this method, a form with the names of behaviors (form number 1) was offered to the evaluator, and he or she marked any behavior of the engagements of a parent with the child. Finally, the number of each behavior was calculated, and in the final form 
Table 1. Characteristics of evaluated samples

\begin{tabular}{|c|c|c|}
\hline Variables & Variable Classes & No.(\%) \\
\hline \multirow{4}{*}{ Age of the child $(y)$} & 3 & $11(19.6)$ \\
\hline & 4 & $14(25)$ \\
\hline & 5 & $17(30.4)$ \\
\hline & 6 & $14(25)$ \\
\hline \multirow[b]{2}{*}{ Gender } & Girl & $21(37.5)$ \\
\hline & Boy & $35(62.5)$ \\
\hline \multirow{3}{*}{ Age of mother $(\mathrm{y})$} & $25-30$ & $12(21.4)$ \\
\hline & $30-35$ & $25(44.6)$ \\
\hline & $35-40$ & 19(33.9) \\
\hline \multirow{3}{*}{ Education degree } & Associate degree & $9(16.07)$ \\
\hline & Bachelor & $31(55.3)$ \\
\hline & MA & $16(28.5)$ \\
\hline
\end{tabular}

Iranian Rehabilitation Journal

(form number 2), the total number of each observed behavior was entered. In the end, data were inserted into SPSS software, and the calculations were performed.

\section{Statistical analysis}

To evaluate the correlation between live observation and video observation, we used the Pearson and Spearman correlation coefficients. Also, to evaluate the correlation between watching of video (inner evaluator validity), we employed both Pearson and Spearman correlation coefficients. The Shapiro-Wilk test was used to evaluate the normal distribution of 10 data. To evaluate the inter-evaluator validation, the Spearman intra-class correlation coefficient was used. Finally, the Kolmogorov-Simonov test was used to evaluate the normality of 56 data.

\section{Results}

The translation period using the Biton method led to the presentation of the Persian version of this test. To evaluate the test's narrative-nominal and understandability, its items were offered to some experts. After studying them and because of the ambiguity of some guidelines, some alterations were applied in these guidelines. For example, guideline number 2 was altered from the statement of "if the communicational texture shows that negative evaluation is done, it is considered the nega- tive talk, even if the stated word is not exactly negative" into "if the face mood, melody, tone, and the sound loudness show that a negative evaluation is performed, it is considered a negative talk." Another example is the third guideline by the statement of "unreal questions which indicate a negative evaluation of characteristics, activities and or child products, is considered as a negative talk." It was changed to "the question sentences that blame the child's behavior or activity with a negative mood."

From the evaluated samples, 35 children were boys $(62.5 \%)$, and most were 5 years old $(30.4 \%)$, and $44.6 \%$ of their mothers were in the age range of 30-35 years, and $55.3 \%$ of their mothers had Bachelor degree (Table 1). Table 2 presents the correlation of evaluations as live and by video on 10 mothers and their children. Table 3 presents the results from the evaluation of validation of the intra-evaluator. The results of validity measurements between evaluators are presented in Table 4 and Table 5, respectively.

\section{Discussion}

As mentioned earlier, the experts provided the Persian version of the DPICS test through translation, adaptation, and face validity. The Pearson correlation coefficient between live and video observation was significant (0.83 to 0.99). Moreover, the results of the Spearman correlation coefficient for the data of this part were significant $(0.74$ to 1$)$. The high correlation coefficient in- 
Table 2. Correlation between live and video observation

\begin{tabular}{|c|c|c|}
\hline Items & Spearman & Pearson \\
\hline Negative talk & --- & 0.98 \\
\hline Direct command & 0.99 & -- \\
\hline Indirect command & 0.99 & --- \\
\hline Labeled praised & --- & 0.99 \\
\hline Non-labeled praise & 0.99 & --- \\
\hline Information questions & 0.99 & --- \\
\hline Descriptive/reflective questions & 0.99 & -- \\
\hline Reflective statement & 0.83 & -- \\
\hline Behavioral descriptive & --- & 0.97 \\
\hline Neutral talk & 0.99 & --- \\
\hline Negative touch and caress & & Unknown \\
\hline Positive touch and caress & --- & 1.000 \\
\hline Answer & --- & 0.91 \\
\hline No answer & --- & 0.99 \\
\hline No opportunity for an answer & & No score recorded \\
\hline Compliance & -- & 0.74 \\
\hline Incompliance & & No score recorded \\
\hline No opportunity for compliance & & No score recorded \\
\hline
\end{tabular}

Iranian Rehabilitation Journa

dicates that encoding, whether live or as videos, has the same conditions for rater and encoding of behaviorslive or video - and does not impact the encoding of behaviors by the rater. These results also indicate a high level of mastery and expertise of the encoder over the test. In similar studies examining the reliability of this test, this method has not been used, so this study is not comparable with other studies in this regard.

Results for Pearson correlation coefficient calculated to verify intra-rater reliability was from 0.87 to 0.99 . Spearman's correlation coefficient, the results for most of the behaviors were significant from 0.89 to 1 ; however, in one of the behavioral items called compliance, a low score was obtained (0.50). One of the reasons for the low correlation of this behavior can be related to the skill and experience of the rater in encoding this behavior. This means that the rater may not be trained enough to encode this behavior. On the other hand, given the abstract nature of this behavior, its encoding was difficult for the rater, leading to less attention and tendency of the rater to encode this behavior compared to other more objective behaviors.

Moreover, the abstract nature of this behavior renders it to be influenced more by the judgment of the rater. This process is the result of the training given, the theory of mind, and the emotional recognition of the raters towards the emotions of mothers during responses to the orders, which could determine conclusions made from the information and their intention in the interpretation of behaviors. Besides all the reasons mentioned above, the passage of time (over a week) may also affect the encoding of this behavior. This means that in the first or second observations of the video, the rater has focused more or less on this behavior, thus making a difference in the encoding of this behavior. Other reasons are the rater's mental and emotional states that may affect the encoding of this behavior. Judging by the results, one- 
Table 3. Correlation between double coding of watching videos (inter evaluator validation)

\begin{tabular}{|c|c|c|}
\hline Questions & Spearman & Pearson \\
\hline Negative talk & --- & 0.99 \\
\hline Indirect command & 0.99 & --- \\
\hline Direct command & 0.99 & --- \\
\hline Labeled praised & --- & 1.00 \\
\hline Non-labeled praise & 0.99 & --- \\
\hline Information questions & 0.99 & --- \\
\hline Descriptive/reflective questions & 0.99 & --- \\
\hline Reflective statement & 0.87 & --- \\
\hline Behavioral descriptive & & 0.96 \\
\hline Neutral talk & 0.99 & --- \\
\hline Negative touch and caress & \multicolumn{2}{|c|}{ No score recorded } \\
\hline Positive touch and caress & --- & 1.00 \\
\hline Answer & --- & 0.89 \\
\hline No answer & --- & 0.92 \\
\hline No opportunity for an answer & \multicolumn{2}{|c|}{ No score recorded } \\
\hline Compliance & --- & 0.50 \\
\hline Incompliance & \multicolumn{2}{|c|}{ No score recorded } \\
\hline No opportunity for an answer & \multicolumn{2}{|c|}{ No score recorded } \\
\hline
\end{tabular}

Iranian Rehabilitation Journal

week time interval and the passage of time have not had much effect on the correct encoding of the behaviors by the encoder, reflecting the command of the rater over encoding behaviors.

The Spearman correlation coefficient and inter-class correlation coefficient were used to assess the reliability of the rater. The results of the Spearman correlation coefficient are high and from 0.81 to 0.99 . The results indicated the excellent mastery, expertise, and experience of the raters in encoding the behaviors. Compared to the study by Eyberg and Besmer (1998) on 20 mothers and children in child-centered and parent-centered positions on DPICS II, the Pearson correlation coefficient was from 0.47 to 0.94 for the child-centered position and 0.25 to 0.99 for the parent-centered position [19-21].

Here 0.47 was related to behavioral descriptive and 0.25 was related to labeled praised, which had low reliabil- ity in their study but we obtained high reliability in these items(behavioral descriptive 0.90 and labeled praised 0.92 ). It should be noted that in the present study, the reliability of these behaviors was calculated in all three positions. That difference with our study may be due to the difference in the cultural factor of the families. Thus, this behavior may have been less seen in Besmer and Eyberg's study, reducing the reliability of these two behaviors. In addition, as Iranian mothers have a rearing style based on emotion [22], they use more admiring words. Also, Iranian mothers are over-supportive; they use behavior description more often and described the child's behaviors more. The expertise and experience of raters in the two studies are another reason for this difference.

Furthermore, Besmer, Brestan, and Eyberg (2005) used DPICS II on 30 mothers and children without the disorder and 30 mothers and children referred for ODD treatment. In that study, they calculated the Pearson cor- 
Table 4. Validity between evaluator by Inter-class Correlation Coefficient (ICC)

\begin{tabular}{|c|c|c|c|}
\hline \multirow{2}{*}{\multicolumn{3}{|c|}{ Questions }} & \multirow{2}{*}{$\frac{\text { Coefficient (ICC) }}{\text { F }}$} \\
\hline & & & \\
\hline \multicolumn{2}{|c|}{ Negative talk (NTA) } & 0.98 & 87.732 \\
\hline \multirow{2}{*}{ Orders } & Direct Command (DC) & 0.99 & 138.683 \\
\hline & Indirect Command (IC) & 0.97 & 62.300 \\
\hline \multirow{3}{*}{ Praised } & Praise (LP) & 0.93 & 27.758 \\
\hline & & & \\
\hline & Non-labeled Praised (UP) & 0.92 & 23.398 \\
\hline \multicolumn{2}{|c|}{ Information Questions (IQ) } & 0.96 & 50.311 \\
\hline \multicolumn{2}{|c|}{ Descriptive/reflective Questions (DQ) } & 0.89 & 16.992 \\
\hline \multicolumn{2}{|c|}{ Reflective Statement (RF) } & 0.89 & 16.796 \\
\hline \multicolumn{2}{|c|}{ Behavioral Descriptive (BD) } & 0.90 & 19.763 \\
\hline \multicolumn{2}{|c|}{ Neutral Talk (TA) } & 0.95 & 42.130 \\
\hline \multirow{2}{*}{ Touch and caress } & Negative Touch and Caress (NTO) & 1.00 & 0.000 \\
\hline & Positive Touch and Caress (PTO) & 0.89 & 17.725 \\
\hline \multirow{3}{*}{ Answer to questions } & Answer (AN) & 0.89 & 17.224 \\
\hline & Non-answer (NA) & 0.934 & 29.128 \\
\hline & No opportunity for answer & 0.79 & 8.361 \\
\hline \multirow{3}{*}{ Answer to questions } & Compliance (CO) & 0.84 & 11.815 \\
\hline & Incompliance (NC) & 0.81 & 9.566 \\
\hline & No opportunity for compliance (NOC) & 0.85 & 12.217 \\
\hline
\end{tabular}

Iranian Rehabilitation Dourna

relation coefficient for all three positions, and the range of correlation coefficient for the parent class was from 0.49 to 0.99 [23]. Reliability of 0.49 is related to positive touch and fondling, which was 0.89 in the present study. This difference can also be attributed to the difference in the experience and mastery of raters in the two research studies and the cultural differences of families that may be less likely to use positive touching and fondling behavior with their children, and this behavior is mainly seen in Iranian families. Also, their study was on two groups with one group having a disorder, the parents use positive touch and fondling less, and parents' behavior is more controlling and restrictive according to the type of disorder they had. Moreover, given the problem in the children, they might also be reluctant to be cuddled and had a positive relationship with their mothers. These factors have affected the incidence of behavior and, conse- quently, the degree of reliability. Another method used to study the inter-rater reliability in this study was the inter-class correlation coefficient. The reliability determined for this type of statistical method is as follows: if the results obtained are from 0 and 0.2 , it is weak; 0.3 to 0.4 , relatively good; 0.5 to 0.6 , good; 0.7 to 0.8 , strong; and more than 0.8 , perfect.

In the present study, the reliability was found between strong and perfect. A high value of the reliability, as in the previous approach, can be attributed to the high level of expertise of the raters in test encoding. In a study by Brestan, Foote, and Eyberg (2005) on DPICS II with 60 fathers and children without and with the referral of ODD disorder, positive contact behaviors (0.48), no answer (0.49), and labeled praised (0.05) had poor reliability, whose results are inconsistent with the results of our study [24]. 
Table 5. Validity between the evaluator by the spearman correlation coefficient

\begin{tabular}{|c|c|}
\hline Items & Spearman Correlation Coefficient \\
\hline Negative talk & 0.95 \\
\hline Direct command & 0.99 \\
\hline Indirect command & 0.97 \\
\hline Labeled praised & 0.92 \\
\hline Non-labeled praise & 0.89 \\
\hline Information questions & 0.93 \\
\hline Descriptive/reflective questions & 0.93 \\
\hline Reflective statement & 0.88 \\
\hline Behavioral descriptive & 0.90 \\
\hline Neutral talk & 0.92 \\
\hline Negative touch and caress & --- \\
\hline Positive touch and caress & 0.89 \\
\hline Answer & 0.89 \\
\hline No answer & 0.88 \\
\hline No opportunity for answer & 0.81 \\
\hline Compliance & 0.91 \\
\hline Incompliance & 0.85 \\
\hline No opportunity for compliance & 0.84 \\
\hline
\end{tabular}

Iranian Rehabilitation Journal

The low reliability of these behaviors can be due to the experience and mastery of raters in encoding this behavior. Regarding positive fondling and touching, one can state that as the parent participating in the study were fathers, they used this behavior less, but in the present study, the parents were mothers, and the maternal emotional style caused the higher use of this behavior. Another reason that might have reduced reliability was the type of disorder. The children with ODD made parents use less positive contact behaviors, and the children were not showing positive fondling (in the present study, the children were normal).

In another study, Bowman (2015) studied DPICS III in 80 healthy and autistic children (40 normal children and 40 autistic children). Some parent and child class behaviors were conducted, and inter-class correlation was used to calculate inter-rater reliability [25]. The results showed good to strong reliability for the parents and the children classes. However, there was an exception: the child's response behavior that received poor (0.48) reliability [25]. In the present study, the parent's class has been investigated but not the child's class.

\section{Conclusion}

In general, it seems that the DPICS test is a good tool for studying the interaction of parents with their children. This study contributes significantly to the history and validity of this test in a healthy population of children. The title of this study was related to the limitation of Bumon's study in 2015, claiming that no studies have been conducted on healthy populations using DPICS III. Besides, the findings of this study could play an essential role in determining interaction disorders between parents and children and a timely indicator to prevent subsequent disorders. Moreover, it brings about the preparation to enter the next steps for validity and reliability studies to examine the psychometric properties of this test in the Persian language. 


\section{Ethical Considerations}

\section{Compliance with ethical guidelines}

The Ethics Committee of the University of Social Welfare and Rehabilitation approved the study. Written informed consent was received from the parents before the program was administered.

\section{Funding}

The study was extracted from the MSc. thesis of the first author at the Department of Speech Therapy, University of Social Welfare and Rehabilitation Sciences, Tehran.

\section{Authors' contributions}

Conceptualization, writing - original draft, and data collection: Sanaz Dehghani Kiadehi; Designing and writing - editing: Fariba Yadegari; Methodology: Akbar Biglarian and Atiyeh Ashtari.

\section{Conflict of interest}

The authors declared no conflict of interest.

\section{Acknowledgments}

We would like to thank the speech-language pathologists and linguists who assisted us with the material validity process, the managers of nursery schools and kindergartens, and the children who took part in the study.

\section{References}

[1] Cox MJ, Harter KSM. Parent-child relationships. In: Bornstein $\mathrm{MH}$, editor. Well-being: Positive development across the life course. Mahwah: Lawrence Erlbaum Associates Publishers; 2003. pp. 191-204. https://books.google.com/ books?id=63Nh4QEmhycC\&dq

[2] Iarocci G, Gardiner E. Social competence during adolescence across cultures. In: Wright JD, editor. International Encyclopedia of the Social \& Behavioral Sciences. $2^{\text {nd }}$ ed. Oxford: Elsevier; 2015. pp. 216-21. [DOI:10.1016/B978-0-08-097086-8.23189-9]

[3] Sanders MR, Turner KMT, Wall CR, Waugh LM, Tully LA. Mealtime behavior and parent-child interaction: A comparison of children with feeding problems, and nonclinic controls1. Journal of Pediatric Psychology. 1997; 22(6):881-900. [DOI:10.1093/jpepsy/22.6.881] [PMID]

[4] Stierlin H. Treatment dilemmas with psychotic and sociopathic patients. British Journal of Medical Psychology. 1963; 36(1):75-84. [DOI:10.1111/j.2044-8341.1963.tb01267.x] [PMID]
[5] Hudson JL, Rapee RM. Parent-child interactions and anxiety disorders: An observational study. Behaviour Research and Therapy. 2001; 39(12):1411-27. [DOI:10.1016/S00057967(00)00107-8][PMID]

[6] Snow CE. Families as social contexts for literacy development. New Directions for Child and Adolescent Development. 1993; 1993(61):11-24. [DOI:10.1002/cd.23219936103]

[7] Vandermaas-Peeler M, Nelson J, Bumpass C, Sassine B. Social contexts of development: Parent-child interactions during reading and play. Journal of Early Childhood Literacy. 2009; 9(3):295-317. [DOI:10.1177/1468798409345112]

[8] Klatte IS, Roulstone S. The practical side of working with parent-child interaction therapy with preschool children with language impairments. Child Language Teaching and Therapy. 2016; 32(3):345-59. [DOI:10.1177/0265659016641999]

[9] Cologon K, Wicks L, Salvador A. Supporting caregivers in developing responsive communication partnerships with their children: Extending a caregiver-led interactive language program. Child Language Teaching and Therapy. 2017; 33(2):157-69. [DOI:10.1177/0265659016650978]

[10] Brady N, Warren S. Language interventions for children with mental retardation. International Review of Research in Mental Retardation. 2003; 27:231-54. [DOI:10.1016/S00747750(03)27007-5]

[11] Konza D, Maloney Grafton P. It takes two to talk: A focused intervention program for parents and children with language delays. The International Journal of Interdisciplinary Social Sciences: Annual Review. 2010; 5(6):225-36 [DOI:10.18848/1833-1882/CGP/v05i06/59301]

[12] Gupta R, Bone D, Lee S, Narayanan S. Analysis of engagement behavior in children during dyadic interactions using prosodic cues. Computer Speech \& Language. 2016; 37:47-66. [DOI:10.1016/j.csl.2015.09.003] [PMID] [PMCID]

[13] Robinson EA, Eyberg SM. The dyadic parent-child interaction coding system: Standardization and validation. Journal of Consulting and Clinical Psychology. 1981; 49(2):245-50. [DOI:10.1037/0022-006X.49.2.245]

[14] Thornberry T, Brestan-Knight E. Analyzing the Utility of Dyadic Parent-Child Interaction Coding System (DPICS) WarmUp Segments. Journal of Psychopathology and Behavioral Assessment. 2011; 33(2):187-95. [DOI:10.1007/s10862-011-9229-6]

[15] Foote R, Eyberg S, Schuhmann E. Parent-child interaction approaches to the treatment of child behavior problems. Advances in Clinical Child Psychology. 1998: 20:125-51. [DOI:10.1007/978-1-4757-9038-2_4]

[16] Awde N. The influence of cultural values on the parentchild interaction patterns of families from an Asian back ground. Arecls. 2009; 6:1-17. https://research.ncl.ac.uk/media/sites/researchwebsites/arecls/awde_vol6.pdf

[17] Ochs E. Culture and language development: Language acquisition and language socialization in a Samoan village. New York: CUP Archive; 1988. https://books.google.com/ books?id=Zwc5AAAAIAAJ\&dq

[18] Beaton DE, Bombardier C, Guillemin F, Ferraz MB Guidelines for the process of cross-cultural adaptation of self-report measures. Spine. 2000; 25(24):3186-91. [DOI:10.1097/00007632-200012150-00014] [PMID] 
[19] Herschell AD, Calzada EJ, Eyberg SM, McNeil CB. Clinical issues in parent-child interaction therapy. Cognitive and Behavioral Practice. 2002; 9(1):16-27. [DOI:10.1016/S10777229(02)80035-9]

[20] Schuhmann EM, Foote RC, Eyberg SM, Boggs SR, Algina J. Efficacy of parent-child interaction therapy: Interim report of a randomized trial with short-term maintenance. Journal of Clinical Child Psychology. 1998; 27(1):34-45. [DOI:10.1207/ s15374424jccp2701_4] [PMID]

[21] Eyberg SM, Nelson MM, Duke M, Boggs SR. Manual for the Dyadic Parent-Child Interaction Coding System Third Edition. 2005. https://www.researchgate.net/publication/242667283_Manual_for_the_Dyadic_Parent-Child_Interaction_Coding_System

[22] Zare H, Tahmassian K, Pakdaman S, Davari Ardakani N, Rahimi T. Observing Iranian Mother-child interaction in a playing situation. Journal of Family Research. 2016; 12(1):725. https://jfr.sbu.ac.ir/article_97250.html?lang=en

[23] Werba BE, Eyberg SM, Boggs SR, Algina J. Predicting outcome in parent-child interaction therapy: Success and attrition. Behavior Modification. 2006; 30(5):618-46. [DOI:10.1177/0145445504272977] [PMID]

[24] Eyberg SM. Dyadic Parent-Child Interaction Coding System (DPICS): Comprehensive manual for research and training. High Springs: PCIT International; 2013. https://books. google.com/books?id=6YwxjgEACAAJ\&dq

[25] Bauman SE. utility of the dyadic parent child interaction coding system with children with autism spectrum disorder: An investigation of reliability and validity [PhD. dissertation]. Mobile: University of South Alabama; 2015. https://www. proquest.com/openview/d23fb87474cd9cbbaca1355ae59abe 8f/1?pq-origsite $=$ gscholar $\& \mathrm{cbl}=18750 \&$ diss $=y$ 